\section{Species diversity regarding the presence of proximal tubular progenitor cells of the kidney}

\author{
J. Hansson, ${ }^{1}$ A.E. Ericsson, ${ }^{2}$ H. Axelson, \\ M.E. Johansson ${ }^{3,4}$ \\ 'Translational Cancer Research, \\ Department of Laboratory Medicine, \\ Medicon Village, University of Lund \\ ${ }^{2}$ Astra-Zeneca R\&D Mölndal, CKD, \\ Bioscience HB258, Mölndal \\ ${ }^{3}$ Center for Molecular Pathology, \\ Department of Translational Medicine, \\ University of Lund \\ ${ }^{4}$ Department of Pathology, Skåne \\ University Hospital, Malmö, Sweden
}

\section{Abstract}

The cellular source for tubular regeneration following kidney injury is a matter of dispute, with reports suggesting a stem or progenitor cells as the regeneration source while linage tracing studies in mice seemingly favor the classical theory, where regeneration is performed by randomly surviving cells. We, and others have previously described a scattered cell population localized to the tubules of human kidney, which increases in number following injury. Here we have characterized the species distribution of these proximal tubular progenitor cells (PTPCs) in kidney tissue from chimpanzee, pig, rat and mouse using a set of human PTPC markers. We detected PTPCs in chimpanzee and pig kidneys, but not in mouse tissue. Also, subjecting mice to the unilateral urethral obstruction model, caused clear signs of tubular injury, but failed to induce the PTPC phenotype in renal tubules.

\section{Introduction}

Upon subtotal acute kidney injury, the normally mitotically almost quiescent kidney displays a robust regenerative capacity, often allowing the tubular integrity to be restored. The cellular origin of renal regeneration is however debated. The classical concept stipulates that randomly surviving tubular cells dedifferentiate and repopulate the tubules. ${ }^{1}$ An alternative hypothesis is that blood bourne or renal stem cells effectuate regeneration. ${ }^{2,3}$ The classical view has lately been added experimental data from linage tracing experiments in mouse, suggesting that regeneration is indeed an intratubular stochastic event and not conducted by a designated subpopulation of cells, such as tubular progenitors. ${ }^{4-7}$ Recent reports have however raised important concerns regarding the interpretation of results from this type of studies. ${ }^{3,8}$ The presence of a distinct scattered tubular cell population in human kidney has recently been described by several groups, including our own. ${ }^{9-14}$ These proximal tubular progenitor cells (PTPCs), ${ }^{13}$ share markers with progenitor cells in the parietal epithelial layer of Bowman's capsule (PEC). ${ }^{12,13}$ The role of PTPCs is not entirely clear at present, but might be to restore the tubular epithelium after acute tubular injury and/or to aid the regeneration process. ${ }^{11,14}$

Various models and species are used to study renal regeneration, and in order to reconcile the discrepant data regarding the basis of tubular regeneration; one potential cause for confusion might be species differences. Herein we have stained chimpanzee, porcine and murine renal tissues for robust markers of the human PTPC, such as low mitochondrial content and expression of Prominin 1 (CD133), Vimentin, Claudin-1 (CLDN1) and Cytokeratins 7 (KRT7) and 19 (KRT19). We have furthermore sought to provoke the expression of PTPC-markers by applying the unilateral urethral obstruction (UUO) model in mouse. Results show that chimpanzee and pig are endowed with cells similar to PTPCs in humans, whereas mice are not, even during renal injury following UUO-modeling.

\section{Materials and Methods}

\section{Procurement of animal tissue}

Renal tissue from a female chimpanzee was obtained from Statens Veterinärmedicinska institut (SVA), Uppsala, Sweden. Renal causes for the death of the animal were excluded on clinical grounds. Normal renal tissue from mouse and rat was a kind gift from Professor Börje Haraldsson, Molecular and Clinical Medicine, Sahlgrenska University Hospital, Gothenburg. Normal healthy porcine tissue was obtained as a kind gift from Professor Stig Steen, Lund University, Sweden.

\section{Immunofluorescence}

Immunofluorescence was performed as previously described. ${ }^{13}$ Murine sections were blocked in $5 \%$ serum $+1 \%$ BSA in phosphate buffered saline $10 \%$ tween (PBST). Chimpanzee and porcine sections were blocked in 3\% bovine serum albumin (BSA), (MP Biomedicals, Santa Ana, CA, USA) in PBST. Choice of antibodies was as far as possible based on previously established stainability in the species of interest. Applied antibodies are presented in Supplementary Table 1. Sections were analyzed by confocal scanning
Correspondence: Martin E. Johansson, Department of Pathology, Skåne University Hospital, Malmö, Jan Waldenströms gata 59, SE20502 Malmö, Sweden.

Tel. +46.40 .337387 - Fax: +46.40 .337063 .

E-mail: martin.johansson@med.lu.se

Key words: Acute tubular necrosis; tubular regeneration; species diversity; proximal tubules.

Contributions: MEJ, study planning, results analysis, manuscript preparation; JH, AEA, HA, study execution, results analysis, manuscript preparation.

Conflict of interest: The authors declared no potential conflicts of interest with respect to the research, authorship, and/or publication of this article.

Acknowledgments: renal tissue from a female chimpanzee was obtained from Statens Veterinärmedicinska institut (SVA), Uppsala. Murine renal tissue was a kind gift from Professor Börje Haraldsson, Molecular and Clinical Medicine, Sahlgrenska University Hospital, Gothenburg. Porcine tissue was a kind gift from Professor Stig Steen, Lund University.

Funding: this study was supported by grants from The Marianne \& Marcus Wallenberg Foundation, the National Association against Kidney Diseases, Professor Lars-Erik Gelins Commemorative fund, Governmental funding of Clinical Research within the National Health Service (ALF), SUS foundations and donations, the Malmö General Hospital Research Fund for cancer research. Gunnar Nilsson foundation. The Strategic Cancer Research Program Biocare and the Swedish Cancer Society.

Received for publication: 17 September 2015. Accepted for publication: 20 December 2015.

This work is licensed under a Creative Commons Attribution-NonCommercial 4.0 International License (CC BY-NC 4.0)

(C) Copyright J. Hansson et al., 2016

Licensee PAGEPress, Italy

European Journal of Histochemistry 2016; 60:2567 doi:10.4081/ejh.2016.2567

using the Zeiss LSM 710 system (Carl Zeiss AG, Jena, Germany).

\section{Animals and unilateral uretheral} obstruction modeling of renal injury

Male C57BL/6NCrl mice were purchased from Charles River Laboratories (Sulzfeld, Germany). Animals received analgesia on the day of surgery and two consecutive days, 0.5 $\mathrm{mg} / \mathrm{kg}$ Temgesic (RB Pharmaceuticals Ltd., Slough, UK). Mice were anesthetized using 
isoflurane and the urether of the left kidney was ligated at two positions, as close to the kidney pelvis as possible. For sham-operated animals, ligatures were immediately removed. Following surgery all mice were weighed daily to keep track of health status. The urethral obstruction was allowed to develop for 5,10 and 14 days ( $n=5$ for UUO and $n=3$ for sham).

\section{Histological evaluation of tissue from the UUO model}

Tissues were fixed in $4 \%$ buffered formaldehyde, dehydrated and embedded in paraffin. Four- $\mu \mathrm{m}$ sections were cut and stained with hematoxylin/eosin according to standard protocol. Adjacent sections were stained for vimentin (ab45939; Abcam, Cambridge, UK) or (AB5733; Millipore, Billerica, MA, USA) as previously described. ${ }^{12}$ The histology was evaluated by an experienced renal pathologist.

\section{Results}

\section{Staining for PTPC-markers in nor-} mal kidney tissue from pig, chimpanzee, mouse and rat

Staining of chimpanzee kidney mirrored the staining pattern previously reported from humans. ${ }^{13}$ Scattered cells positive for the PTPC-markers vimentin, CLDN1, KRT7, KRT19 and CD133 could be detected within proximal tubules (Figure 1 A-D). The markers were also present in the PEC layer of Bowman's capsule, in line with the human setting. Moreover, using the mitochondrial marker MTC02, vimentin positive cells also demonstrated lower mitochondrial content than the surrounding PT cells (Figure 1E), a hallmark of human PTPCs. ${ }^{13}$

In porcine tissues, vimentin staining identified scattered PT cells (Figure 1F), that also displayed a lower mitochondrial content than adjacent PT cells (Figure 1J). This was found to be the case in two separate strains of pigs. However, no co-localization was seen with other PTPC markers using the available antibodies (Figure 1 G-I). CLDN1 antibody stained PEC however, indicating that the antibody was suitable for staining porcine tissue (Figure 1I). KRT7 failed to stain cells in PTs, instead apical staining of the distal tubules was seen, providing a positive control for the staining (Figure $1 \mathrm{G}-\mathrm{H}$ ). In contrast, in renal tissue from mouse none of the above markers could identify scattered cells within PTs, as exemplified by vimentin that stained interstitial cells, PECs and glomeruli as expected, whereas no positive tubular cells were detected (Figure 2A). Furthermore KRT7, KRT19, CLDN1 and CD133 also failed to identify a PTPC popula- tion. KRT7 staining was localized to distal tubules and collecting ducts as expected, serving as internal control (Figure 2D). Finally, in mice no cells with paucity of mitochondrial staining were detected. In rat only one out of three rat compatible vimentin antibodies displayed any positive staining in PTs (Figure 2 B). However, the frequency of vimentin posi-
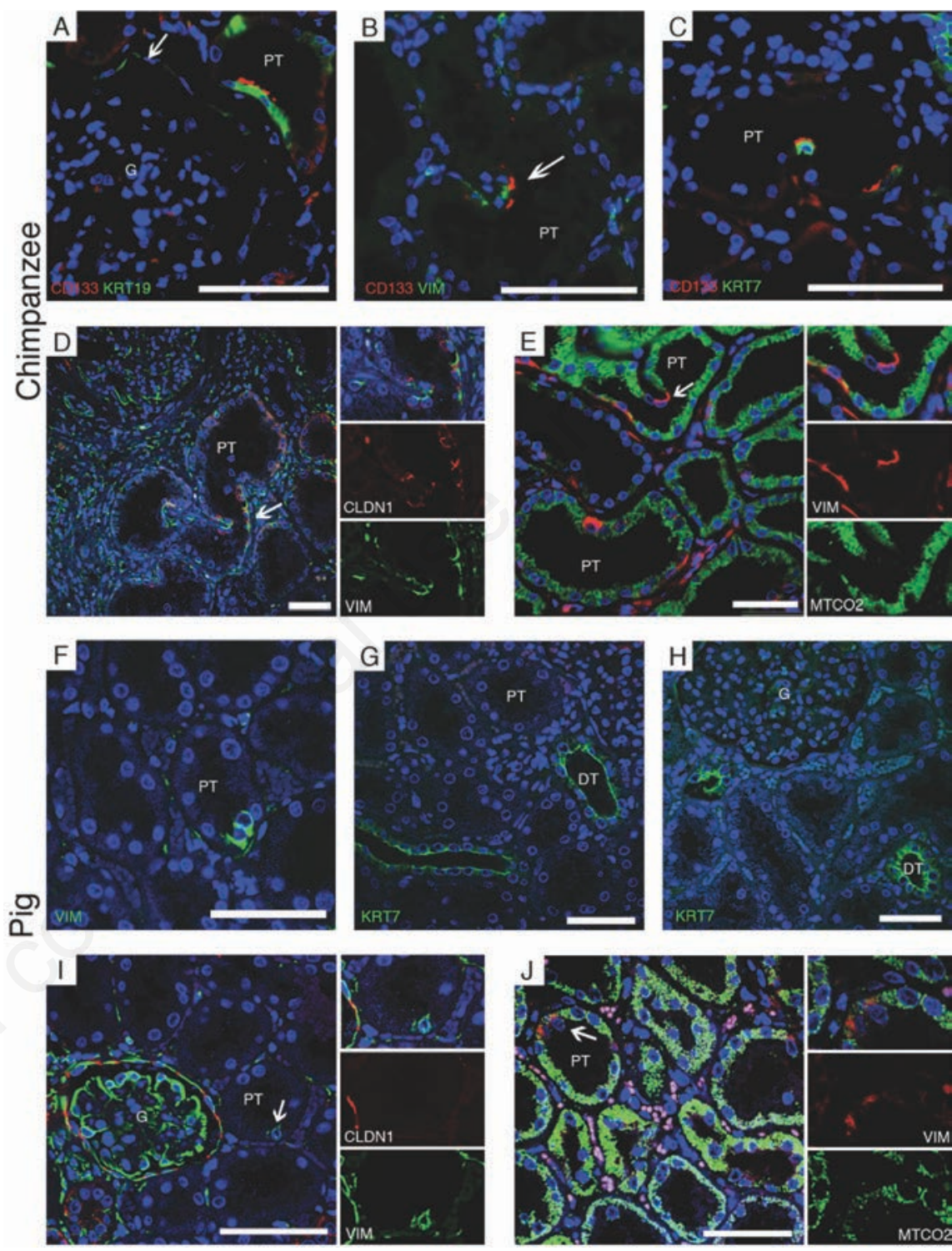

Figure 1. PTPCs present in renal cortex of chimpanzee and pig. A-E) Immunofluorescent (IF) staining for PTPC-markers using renal tissue from chimpanzee. A) KRT19 showed positive staining of PECs of Bowman's capsule (arrow) and co-localization with CD133 in scattered PT cells. B) Vimentin co-localization with CD133 in duplet (arrow) in PT. C) Co-staining of KRT7 and CD133 identifies single scattered PT cells. D) Scattered PT cells are co-positive for CLDN1 and vimentin. E) Vimentin positive cells had lower or no mitochondrial staining as assessed by MTCO2. F-J) IF staining with PTPC markers on porcine renal tissue. F) Vimentin staining in PT show vimentin positive cell duplet, replicating results seen in human. G-H) KRT7 staining showed positive cells in distal tubules, while no positive PT cells were detected. I) Vimentin co-localization with CLDN1 showed positive parietal epithelial cells in Bowman's capsule, while only vimentin proved positive in scattered cells in PT. J) Co-staining of vimentin and mitochondrial marker MTCO2, showed vimentin positive cells with low mitochondrial staining; arrows indicate area of interest shown at a higher magnification in the right panel. Scale bars: A-C) $100 \mu \mathrm{m}$; DJ) $50 \mu \mathrm{m}$. 
tive cells was drastically lower compared to human samples. Additionally, co-staining with vimentin and the mitochondrial protein VDAC1 revealed that some of the vimentin positive PT cells appeared to have lower mitochondrial content (Figure 2C), much like the cells in human. In rat the human PTPC-marker KRT7 showed positive staining in distal tubules and collecting ducts, providing an internal positive control, while no positive PT cells were seen (Figure 2E). PECs of Bowman's capsule of stained positive for CLDN1 whereas proximal tubules were negative (Figure 2F).

\section{Staining for PTPC in mouse kidneys subjected to UUO}

Control kidneys stained with hematoxylin/ eosin showed ordinary histology, with no tubulointerstitial injury (Figure 3A). Vimentin staining only showed expected positivity in podocytes, PECs and mesenchymal cells (Figure $3 \mathrm{~B}$ ). Subjecting mice to 14 days of UUO, resulted in clear changes in renal morphology. Histological signs of progressive renal injury could be seen, as interstitial edema and tubular injury with reduced tubular epithelial height and concomitant interstitial fibrosis (Figure 3C). Despite these findings there were no signs of intratubular vimentin positivity (Figure 3D). However an increased staining of interstitial cells could be appreciated, probably indicating an influx of macrophages and increased presence of myofibroblasts associated with injury.

\section{Discussion}

We have investigated the presence of PTPCs in a set of animal species representing important aspects of biology, such as longevity, size and frequency of use as model systems for research. We demonstrate a species difference in the occurrence of these cells and show that cells with the characteristic PTPC features, vimentin positivity and mitochondrial paucity can be found in kidney tissue from chimpanzee and pig in a distribution similar to humans, but not in mice. Moreover, chimpanzee completely mirrored the human PTPC staining pattern. In rat, vimentin positive cells were infrequent and vim ${ }^{+} /$mitochondria $^{\text {low }}$ even rarer, making the findings hard to interpret. Mouse models are important tools for contemporary science. The lack of PTPC-marker staining in normal mouse tissues is therefore problematic, since species variation might be at hand. The lack of these markers in the PT of mice does not exclude that such cells exist, but may suggest that they may express another set of markers. Importantly, no mitochondrial paucity could be detected in normal mouse proximal tubules. Since it has been reported that cells similar to PTPCs can be induced by use of the UU0-model in rats, ${ }^{9}$ we investigated if UUO in mice could induce a PTPC phenotype of similar distribution in mouse tubules. The UUO model resulted in expected changes of kidney morphology and histology, with signs of tubular cellular injury and initial signs of renal fibrosis. However, no PTPC induction could be detected in the tubules. Since mouse is very
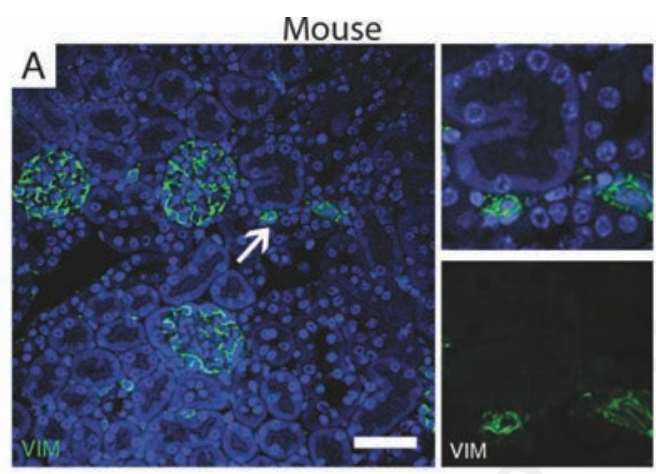

Rat
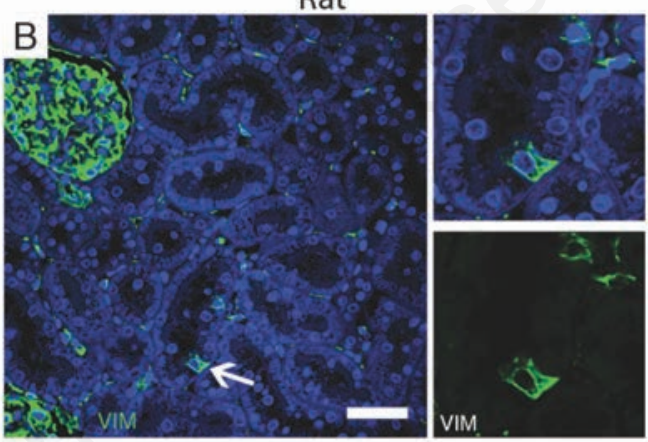

Rat
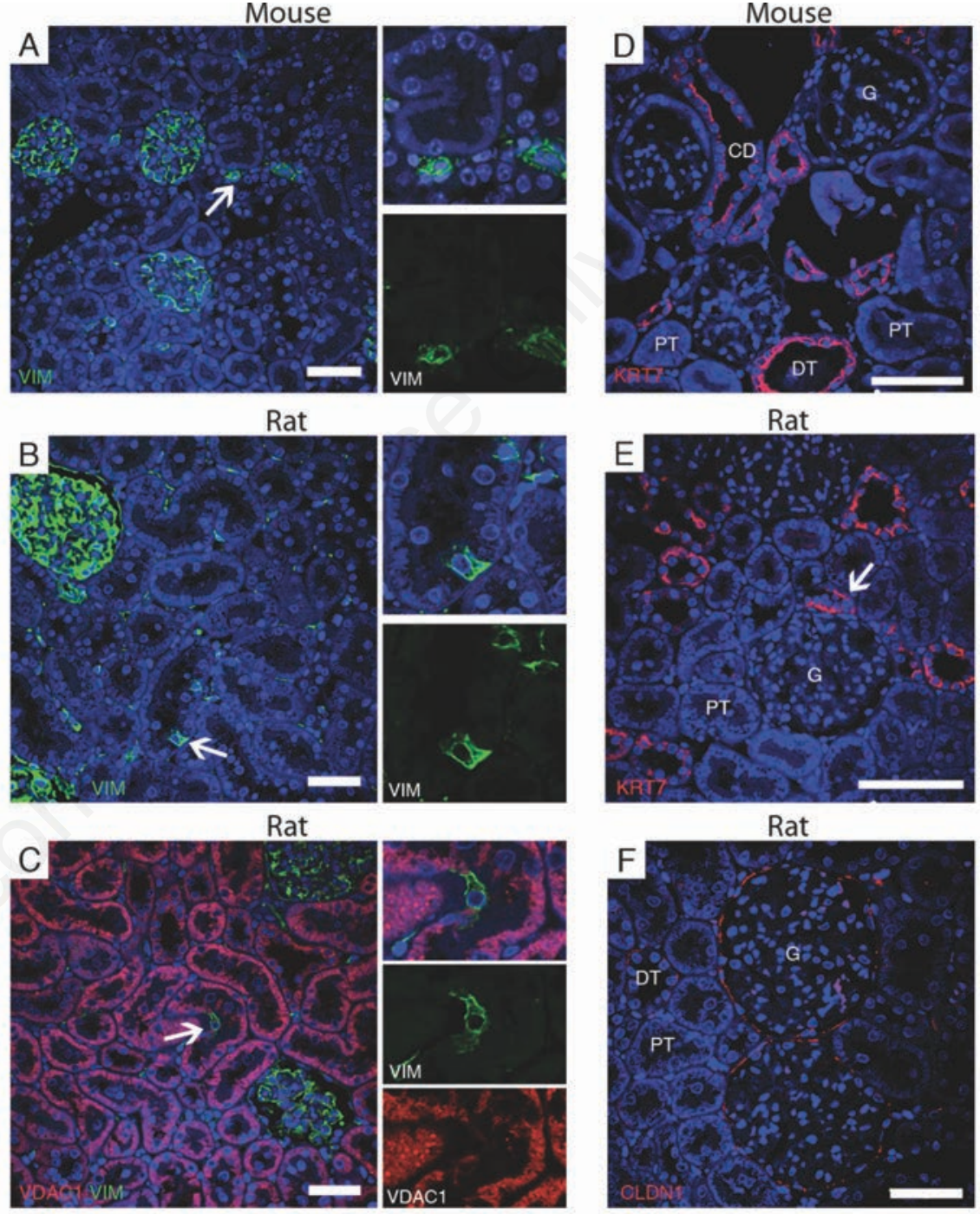

Figure 2. Mouse kidneys are negative for human PTPC-markers, whereas a low frequency of vimentin positive mitochondrial low cells is found in rat. A-F) IF stainings for PTPCmarkers in murine tissue. A) Vimentin staining of mouse renal kidney cortex shows positive glomeruli and interstitial cells, (arrow) while no positive PT cells were detected. B) Rat tissue displayed sparse vimentin positive PT cells (arrow), however only one out of three antisera gave this result. C) Co-staining of vimentin and the mitochondrial marker VDAC1 show vim positive cells with lower VDAC1 intensity. D-E) KRT7 staining of mouse and rat kidney cortex show positive cells in distal tubules and collecting ducts as expected, including the macula densa (arrow). No positive PT cells were detected. F) Staining of cortical rat kidney tissue for the human PTPC marker CLDN1 showed positivity in PECs of Bowman's capsule surrounding the glomerulus, while no positive PT cells were detected. Inserts show single positive cells at a higher magnification. G, glomerulus; PT, proximal tubule; DT, distal tubule; CD, collecting duct. Scale bars: AC,G) $50 \mu \mathrm{m}$; D-F) $100 \mu \mathrm{m}$. 
recta, in male mice. ${ }^{15}$ The cells were characterized by positive staining for vimentin, but in contrast to human PTPCs the cells had abundant mitochondria. These mitochondrion high cells transiently increased in numbers following UUO, but were subsequently lost, due to atrophy after a few days. Whether these cells are functionally similar to human PTPCs is not clear. Being similar in shape, they dramatically
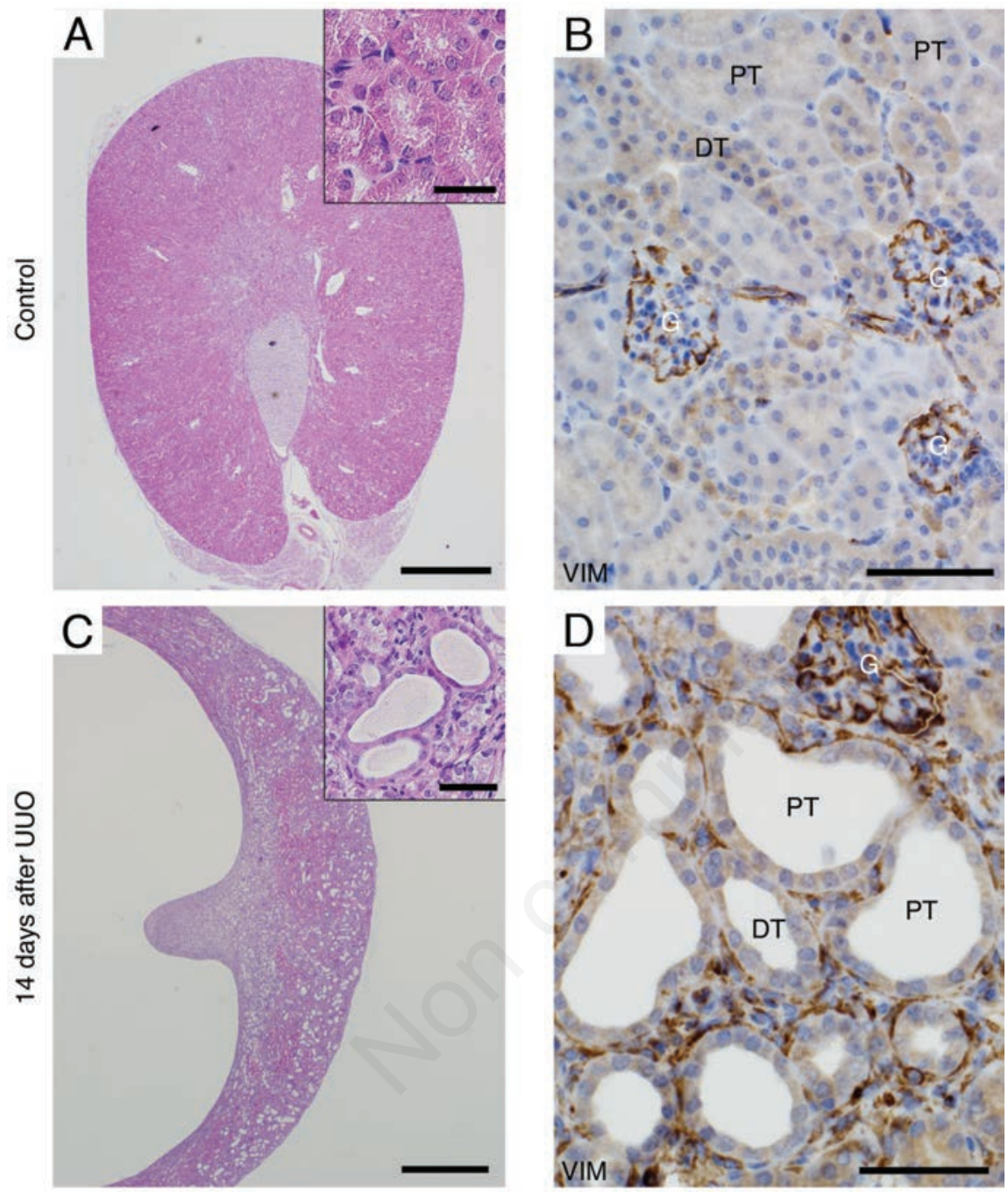

Figure 3. No induction of PTPC in mouse following unilateral urethral obstruction (UUO). A-D) UUO modeling of renal injury in mouse. A) Hematoxylin eosin (H\&E) staining of control kidney showing normal kidney histology. Insert of higher magnification shows normal tubules with ordinary epithelial height and no signs of interstitial fibrosis. B) Vimentin staining of normal kidney, staining is detected in podocytes and endothelial cells as expected, serving as positive control; no tubular cells show any staining. C) H\&E staining of mouse kidney subjected to 14 days UUO; the kidney is severely dilated due to the urethral obstruction with resultant cortical compression and severe reduction of medulla; insert shows tubular atrophy and dilation, as well as interstitial fibrosis and edema. D) Vimentin staining of mouse kidney subjected to 14 days of UUO; apart from the normal vimentin positive structures described above, an additional signal is seen in the intersitial compartment, where the vimentin signal is increased probably due to the increased presence of myofibroblasts and fibroblasts secondary to injury; no intratubular cells are stained however. G, glomerulus; P, Podocyte; PT, proximal tubule; DT, distal tubule. Scale bars 100: $\mu \mathrm{m}$. In B and D, hematoxylin is used as nuclear counterstain. source of energy. ${ }^{17}$ Low mitochondrial content being a key characteristic of the human PTPCs, this most likely suggests that the morphologically distinct cells in pars recta of the mouse kidney have a different biological role.

Due to the characteristic PTPC vimentin expression, it has been argued that presence of these scattered cells in adult human is merely a sign of cellular injury. ${ }^{9,18}$ However, we have recently shown that these cells exist also in healthy children from the ages 2 and up ${ }^{13}$ indicating that the PTPC phenotype in humans is a normal finding. Additionally, PTPCs and PEC have a high degree of similarity in marker composition. Regardless of ones view on this issue, the PECs can hardly be regarded as constantly injured cells and the similarities require further investigation. Undoubtedly, renal regeneration in mice occurs without the aid of PTPCs with human characteristics. Since the mouse nephron is approximately 8 mm long, ${ }^{19}$ compared to the $3 \mathrm{~cm}$ of the human counterpart, one might speculate that the size and length difference of the nephrons could play a role in the distribution of and need for these cells. The lack of PTPCs in tubular epithelium of uninjured mouse kidney could suggest that renal regeneration might be orchestrated in a species-specific manner. These findings may have implications for understanding tubular regeneration in different species and hence the interpretation of data from renal injury model systems.

\section{References}

1. Bonventre JV. Dedifferentiation and proliferation of surviving epithelial cells in acute renal failure. J Am Soc Nephrol 2003;14:S55-61.

2. Little MH. Regrow or repair: potential regenerative therapies for the kidney. $\mathrm{J}$ Am Soc Nephrol 2006;17:2390-401.

3. Lombardi D, Becherucci F, Romagnani P. How much can the tubule regenerate and who does it? An open question. Nephrol Dial Transplant 2015;pii: gfv262.

4. Humphreys BD, Czerniak S, Dirocco DP, Hasnain W, Cheema R, Bonventre JV. Repair of injured proximal tubule does not involve specialized progenitors. Proc Natl Acad Sci USA 2011;108:9226-31.

5. Humphreys BD, Valerius MT, Kobayashi A, Mugford JW, Soeung S, Duffield JS, et al. Intrinsic epithelial cells repair the kidney after injury. Cell Stem Cell 2008;2:284-91.

6. Berger K, Bangen JM, Hammerich L, Liedtke C, Floege J, Smeets B, et al. Origin of regenerating tubular cells after acute kidney injury. Proc Natl Acad Sci USA 2014;111:1533-8. 
7. Kusaba T, Lalli M, Kramann R, Kobayashi A, Humphreys BD. Differentiated kidney epithelial cells repair injured proximal tubule. Proc Natl Acad Sci USA 2014;111:1527-32.

8. Romagnani P, Rinkevich Y, Dekel B. The use of lineage tracing to study kidney injury and regeneration. Nat Rev Nephrol 2015;11:420-31.

9. Smeets B, Boor P, Dijkman H, Sharma SV, Jirak P, Mooren F, et al. Proximal tubular cells contain a phenotypically distinct, scattered cell population involved in tubular regeneration. J Pathol 2013;229:645-59.

10. Sallustio F, De Benedictis L, Castellano G, Zaza G, Loverre A, Costantino V, et al. TLR2 plays a role in the activation of human resident renal stem/progenitor cells. FASEB J 2010;24:514-25.

11. Sallustio F, Costantino V, Cox SN, Loverre A, Divella C, Rizzi M, et al. Human renal stem/progenitor cells repair tubular epithelial cell injury through TLR2-driven inhibin-A and microvesicle-shuttled decorin. Kidney Int 2013;83:392-403.

12. Lindgren D, Bostrom AK, Nilsson K, Hansson J, Sjolund J, Moller C, et al. Isolation and characterization of progenitor-like cells from human renal proximal tubules. Am J Pathol 2011;178:828-37.

13. Hansson J, Hultenby K, Cramnert C, Ponten F, Jansson H, Lindgren D, et al. Evidence for a morphologically distinct and functionally robust cell type in the proximal tubules of human kidney. Hum Pathol 2014;45:382-93.

14. Angelotti ML, Ronconi E, Ballerini L, Peired A, Mazzinghi B, Sagrinati C, et al. Characterization of renal progenitors committed toward tubular lineage and their regenerative potential in renal tubular injury. Stem Cells 2012;30:1714-25.
15. Forbes MS, Thornhill BA, Galarreta CI, Chevalier RL. A population of mitochondrion-rich cells in the pars recta of mouse kidney. Cell Tissue Res 2016;363:791-803.

16. Qian W, Nishikawa M, Haque AM, Hirose M, Mashimo M, Sato E, et al. Mitochondrial density determines the cellular sensitivity to cisplatin-induced cell death. Am J Physiol Cell Physiol 2005;289: C1466-75.

17. Rehman J. Empowering self-renewal and differentiation: the role of mitochondria in stem cells. J Mol Med 2010;88:981-6.

18. Kusaba T, Humphreys BD. Controversies on the origin of proliferating epithelial cells after kidney injury. Pediatr Nephrol 2014;29:673-9.

19. Zhai XY, Thomsen JS, Birn H, Kristoffersen IB, Andreasen A, Christensen EI. Three-dimensional reconstruction of the mouse nephron. J Am Soc Nephrol 2006;17:77-88. 